\title{
Efectos terapéuticos del vendaje neuromuscular en parálisis cerebral infantil: una revisión sistemática
}

\author{
Therapeutic effects of kinesio taping in children with cerebral \\ palsy: a systematic review
}

\author{
Lic. en Fisioterapia Joaquín Ortiz Ramírez y Dra. Sagrario Pérez de la Cruz
}

\begin{abstract}
RESUMEN
La parálisis cerebral infantil es una enfermedad neurológica no progresiva. Es una de las causas más comunes de discapacidad en niños. Son numerosas las técnicas de terapia física que se emplean en la actualidad para su tratamiento; el vendaje neuromuscular es una de ellas. El objetivo principal es revisar los resultados logrados por el vendaje neuromuscular en los estudios científicos publicados en pacientes pediátricos con parálisis cerebral y determinar la calidad metodológica de ellos.

Se revisaron las principales bases de datos científicas, al igual que los estudios publicados en la página oficial de la Asociación Española de Vendaje Neuromuscular.

Se admitieron nueve estudios, que han aportado resultadosimportantes. Estos estudios muestran la efectividad para recuperar funcionalidad en el miembro superior, problemas de deglución y funcionalidad motora que estos pacientes pudieran presentar, aunque la evidencia científica que mostraron puede incrementarse con mejoras en su metodología.

Palabrasclave:vendajeneuromuscular, recuperación de la función, parálisis cerebral, pediatría, deglución.

http:/ / dx.doi.org/10.5546/ aap.2017.e356

Texto completo en inglés:

http:/ / dx.doi.org/10.5546/aap.2017.eng.e356
\end{abstract}

a. Facultad de

Ciencias de la Salud, Universidad de

Almería.

La Cañada de San

Urbano (Almería),

España.

Correspondencia:

Dra. Sagrario Pérez de

la Cruz: spd205@ual.es

Financiamiento:

Ninguno que declarar.

Conflicto de intereses:

Ninguno que declarar.

Recibido: 2-3-2017

Aceptado: 27-6-2017
Cómo citar: Ortiz Ramírez J, Pérez de la Cruz S. Efectos terapéuticos del vendaje neuromuscular en parálisis cerebral infantil: una revisión sistemática. Arch Argent Pediatr 2017;115(6):e356-e561.

\section{INTRODUCCIÓN}

La parálisis cerebral infantil (PCI) es una enfermedad neurológica no progresiva, que se produce en un cerebro inmaduro, y es una de las causas más comunes de discapacidad en niños. ${ }^{1}$ Como consecuencia, se producen alteraciones posturales y de integración sensorial ${ }^{2}$ debido a problemas con el tono muscular, el equilibrio, trastornos de la coordinación y debilidad muscular. Todo ello influye sobre la funcionalidad motora del niño y de su independencia. ${ }^{1}$ También pueden verse asociadas alteraciones cognitivas, de comportamiento, de la sensibilidad, epilepsia y problemas de percepción, problemas audiovisuales, incontinencia urinaria y estreñimiento, ${ }^{3}$ que conducen a restricciones en su capacidad para realizar actividades básicas de la vida diaria (ABVD). ${ }^{3-5}$

Son numerosas las técnicas de terapia física que se emplean en la actualidad para el tratamiento de las alteraciones motoras y no motoras presentadas por estos pacientes. Son tratamientos comunes la toxina botulínica, férulas seriadas, cirugía ortopédica y / u ortesis. Estas intervenciones, algo invasivas, están diseñadas para actuar a nivel periférico, sin pretender promover el desarrollo motor más normal desde el punto de vista central. Otra de las terapias con las que se puede contar en la actualidad es el vendaje neuromuscular (VNM) o kinesiotape.

El VNM (kinesio tape o kinesiotape en inglés) es el nombre que recibe la venda adhesiva creada por Kenzo Kase en Japón, en $1973 .{ }^{6}$ En los últimos años, el concepto de VNM se ha extendido ampliamente y fue diseñado para imitar las propiedades elásticas de la piel humana (igualdad en el peso y en el grosor).

El vendaje está compuesto en su totalidad de algodón, con una capa de pegamento antialérgica, que permite la evaporación y el secado rápido. Estas propiedades le otorgan una resistencia que permite su aplicación 
por un tiempo prolongado, generalmente, tres o cinco días, e, incluso, estar en contacto con el agua. El esparadrapo es elástico hasta un $140 \%$ e iguala la elasticidad de la piel. Las diferentes formas de aplicar el esparadrapo son las siguientes: técnica en I (por encima del vientre muscular), técnica en $\mathrm{Y}$ (alrededor del vientre muscular), técnica en $X$ (desde un punto central alrededor del vientre muscular), técnica en pulpo (para drenaje linfático), técnica en donut (para aumentar espacio) o técnica en estrella (para aumentar espacio en el centro). ${ }^{6,7}$

El creador de esta técnica ha descrito efectos terapéuticos, que dependerán tanto de la cantidad de estiramiento a la que sea sometida la cinta como de la dirección de su aplicación. ${ }^{7,8}$ Dada la posibilidad de aplicarlo en cualquier músculo o articulación del cuerpo, ${ }^{9}$ y conociendo las posibles influencias en la regulación del tono muscular, la indicación de este vendaje está siendo aplicada en el ámbito deportivo para mejorar el rendimiento y/o prevención de lesiones. ${ }^{10,11}$ Igualmente, está resultando de utilidad en el campo de la traumatología, de la ortopedia y de la actividad deportiva. En los últimos años, están apareciendo nuevas aplicaciones en el campo de la neurología, ${ }^{12-14}$ la reumatología ${ }^{15,16}$ e, incluso, la uroginecología, ${ }^{17,18}$ se revelan efectos que, hasta la fecha, no habían sido descritos (como mejorar la retroalimentación sensorial) y que aún deben ser demostrados por la evidencia científica. De igual modo, se vienen realizando estudios comparativos entre los efectos del VNM y otras modalidades de vendaje ya establecidas desde hace años. ${ }^{19,20}$

Sin embargo, y pese a su popularidad, existen pocos estudios científicos que sustenten el uso de este tipo de vendaje. Aún son controvertidos los efectos que se le atribuyeron en su día y no hay suficiente evidencia científica que avale los logros que se defienden, como puede ser el efecto tonificante o relajante en el incremento de la estimulación de mecanorreceptores, efecto sobre el tejido fascial, en la reducción de la presión debajo de la piel, al facilitar el flujo sanguíneo en áreas de dolor, efecto antiinflamatorio o antiedematoso por su acción en los receptores exteroceptivos y propioceptivos. ${ }^{21}$

Lo que sí se está defendiendo es que el VNM en conjunto con otras intervenciones terapéuticas puede promover la integración del proceso de rehabilitación, aumentar la independencia de las ABVD y mejorar su calidad. ${ }^{22}$

El objetivo principal de este trabajo consiste en revisar los resultados logrados por el VNM en los estudios científicos publicados en pacientes pediátricos con PCI y determinar la calidad metodológica de ellos.

\section{METODOLOGÍA}

Se ha realizado una revisión bibliográfica en las bases de datos Medline, Ovid, Cinahl, Pedro, Scopus, The Cochrane Library, EMBASE, Science Direct, Índice Médico Español (IME), así como la página web oficial de la Asociación Española de Vendaje Neuromuscular (AEVNM).

Las palabras clave empleadas fueron kinesiotape, kinesio tape, tape, kinesiotaping, taping, VNM, cerebral palsy, cerebral palsy physical therapy, cerebral palsy children. Se realizaron truncamientos para incluir posibles variaciones utilizadas en la literatura. Esos términos fueron combinados entre sí.

Se han aplicado varios límites en las bases de datos, como publicaciones escritas en inglés, francés, portugués o español y cuyos participantes sean humanos. Asimismo, se ha optado por no delimitar el intervalo de tiempo, ya que este tema lleva varios años estudiándose en países de habla inglesa, aunque, en España, sea un tema

TABLA 1. Criterios de inclusión y exclusión

\begin{tabular}{|c|c|}
\hline CRITERIOS DE INCLUSIÓN & CRITERIOS DE EXCLUSIÓN \\
\hline $\begin{array}{l}\text { - Idiomas: inglés, castellano, francés y portugués. } \\
\text { - Período de publicación: sin límite de tiempo. } \\
\text { - Características de la población: sujetos con diagnóstico } \\
\text { de parálisis cerebral infantil. } \\
\text { - Tipo de intervención: empleo del kinesio tape en } \\
\text { el tratamiento físico, aislado o asociado con otros } \\
\text { tratamientos no quirúrgicos. } \\
\text { - Estudios originales (cualitativos, cuantitativos, combinados), } \\
\text { revisiones bibliográficas y estudios de un único caso. }\end{array}$ & $\begin{array}{l}\text { - Literatura gris y editoriales. } \\
\text { - Participantes con enfermedades asociadas que } \\
\text { interfieran en los resultados del estudio. } \\
\text { - Artículos que presentan intervenciones quirúrgicas que } \\
\text { distorsionen los resultados de este como alternativa } \\
\text { de tratamiento. }\end{array}$ \\
\hline
\end{tabular}


emergente aún, y no perder cualquier tipo de estudio que resulte valioso para esta revisión (criterios de inclusión y exclusión, Tabla 1).

Además, se han realizado búsquedas manuales en las revistas electrónicas consideradas de mayor relevancia para el asunto por estudiar. También se han realizado búsquedas con la técnica "bola de nieve" (snowballing), revisando las listas de referencias de los artículos ya incluidos para revisión en este trabajo para verificar la existencia de artículos adicionales no emergentes en bases de datos.

Para analizar la validez interna de los estudios, se ha utilizado el epígrafe A (preguntas de eliminación y de detalle) de la guía CASPe para la lectura crítica de un ensayo clínico (Tabla 2). La guía está constituida por once preguntas agrupadas en tres bloques. Permite, de forma rápida, obtener información útil sobre las características metodológicas de los estudios, lo que propicia el análisis crítico de estos. La puntuación obtenida por cada uno de los estudios seleccionados muestra la calidad presentada en ellos. Según el tipo de estudio (en nuestro caso, tratamiento), se encuentra un nivel de evidencia que va desde I (formado por ensayos clínicos aleatorizados -ECA- y metaanálisis), II (estudios de cohortes y ECA de baja calidad), III (estudios de casos y controles), IV (series de casos, estudios de cohortes o de casos y controles de baja calidad) y V (opinión de expertos).

Se ha contado también con la escala Jadad para la evaluación de la calidad en un ensayo clínico; su máxima puntuación es 5 (máxima calidad metodológica), y la mínima, 0. Las preguntas se centran, principalmente, en la evaluación de la validez interna del estudio, la relevancia de los resultados y la utilidad práctica, ya que se debe evaluar la validez de los resultados del estudio, junto con su importancia clínica y su factibilidad. Todas las preguntas ofertan respuestas afirmativas, intermedias o negativas. Las respuestas negativas restan validez y aplicabilidad a los resultados, así como a las recomendaciones que proponen los investigadores (Tabla 3).

\section{RESULTADOS}

El total de artículos encontrados han sido 480 en las diferentes bases de datos y a través de la AEVNM. Respecto al año de publicación, en los últimos años, se puede observar un incremento importante de estudios sobre el VNM; los años 2012 y 2014 son los de mayor publicación.

Se encontraron 15 publicaciones potencialmente válidas por estar referidas a la aplicación de esta técnica en el campo de la neurología, pero sin especificar. Después de la aplicación de los criterios de selección, la calidad metodológica de los estudios incluidos y del análisis detallado de estos, se aceptaron un total de siete artículos al cumplir plenamente los criterios de inclusión descritos con anterioridad.

En la Tabla 3, se muestra un resumen de los principales estudios aceptados en esta revisión.

\section{TABLA 2. Epígrafes que componen la guía CASPe}

Epígrafe A: ¿Son válidos los resultados del ensayo?

Preguntas de eliminación:

- ¿Se orienta el ensayo a una pregunta claramente definida?

- ¿Fue aleatoria la asignación de los pacientes a los tratamientos?

- ¿Fueron adecuadamente considerados hasta el final del estudio todos los pacientes que entraron en él?

Preguntas de detalle:

- ¿Se mantuvieron ciegos al tratamiento los pacientes, los clínicos y el personal del estudio?

- ¿Fueron similares los grupos al comienzo del ensayo?

- Al margen de la intervención en estudio, ¿los grupos fueron tratados de igual modo?

Epígrafe B: ¿Cuáles son los resultados?

- ¿Es muy grande el efecto del tratamiento?

- ¿Cuál es la precisión de ese efecto?

Epígrafe C: ¿Pueden ayudarnos estos resultados?

- ¿Pueden aplicarse estos resultados en tu medio o población local?

- ¿Se tuvieron en cuenta todos los resultados de importancia clínica?

- ¿Los beneficios que se van a obtener justifican los riesgos y los costes? 


\section{DISCUSIÓN}

No existe una gran variedad de estudios realizados sobre el VNM para el tratamiento de pacientes con PCI. Los estudios sobre su eficacia se están realizando en estos últimos años (20062016).

De los siete artículos incluidos en esta revisión, dos intentan demostrar la eficacia del VNM en la funcionalidad motora de niños con PCI; ${ }^{23,24}$ otros dos fijan sus objetivos en medir la eficacia en la mejora de la sialorrea de estos niños, ${ }^{25,26}$ uno trata sobre el efecto que provoca en la funcionalidad de la mano, ${ }^{27}$ uno habla sobre el efecto que provoca el VNM sobre el genu recurvatum ${ }^{28}$ que algunos niños con PCI padecen y, por último, otro artículo intenta medir la eficacia en la funcionalidad del miembro superior en estos niños. ${ }^{29}$

Los dos estudios que centran su investigación en medir la eficacia del VNM en la funcionalidad motora en niños con PCI no obtienen resultados estadísticamente significativos. Tanto Kaya Kara O et al., ${ }^{23}$ como Simsek TT et al., ${ }^{24}$ usaron un grupo control y un grupo experimental. La forma de evaluar los resultados fue similar: ambos utilizaron la escala de independencia funcional en la infancia (WeeFIM) y la escala Gross Motor Function Measure (GMFM). Simsek TT et al., ${ }^{24}$ también evaluaron la posición en sedestación con la escala SAS y encontraron diferencias significativas con respecto a la alineación corporal. Las dos investigaciones utilizaron una muestra y una duración similares. En ambos estudios, se encontraron mejorías, aunque no se cumplieron los objetivos propuestos. El principal problema de no encontrar unos resultados que apoyaran la eficacia de este tipo de vendaje fue el poco tiempo dedicado al estudio, ya que ambos estudios fueron realizados durante un período de tres meses. Es necesario que los futuros estudios amplíen su muestra y su duración, ya que la rehabilitación de estos pacientes, a veces, es lenta y se necesita tiempo para poder evaluar si el VNM es de ayuda o no.

Otra de las dificultades que presentan estos niños es la sialorrea, debido a que la salivación excesiva dificulta su alimentación y el habla, ${ }^{25} \mathrm{y}$ ello conlleva diferentes problemas psicosociales. Supone un problema en la calidad de vida de estos pacientes. El VNM es aplicado en los músculos anteriores del cuello y faciales, lo que ayuda a recobrar el control motor, fortalecer el músculo y asistir la elevación laríngea. Autores como Caneschi WF et al., ${ }^{25}$ y Tello CL et al., ${ }^{26}$ encuentran diferencias significativas en sus resultados, por

TABLA 3. Tabla-resumen de los artículos seleccionados

\begin{tabular}{|c|c|c|c|c|c|c|}
\hline Autor/es & $\begin{array}{l}\text { Año de } \\
\text { ublicación }\end{array}$ & Objetivo & $\begin{array}{l}\text { Muestra } \\
\text { Edad } \\
\text { en años }\end{array}$ & $\begin{array}{l}\text { Distribución } \\
\text { de la muestra }\end{array}$ & $\begin{array}{r}\text { (gt } \\
\text { esca }\end{array}$ & $\begin{array}{c}\text { Calidad } \\
\text { (guía CASPe)/ } \\
\text { escala de JADAD }\end{array}$ \\
\hline Keklicek H, et al. ${ }^{27}$ & 2015 & $\begin{array}{l}\text { Evaluar el efecto sobre } \\
\text { la función de la mano }\end{array}$ & $\begin{array}{c}45 \\
\text { Edad: } 4-14\end{array}$ & $\begin{array}{l}2 \text { grupos. } \\
\text { G1: evaluado con y } \\
\sin K T ; G 2: \sin K T\end{array}$ & Efectivo & $\mathrm{II} / 3+$ \\
\hline Kaya Kara O, et al. ${ }^{23}$ & 2014 & $\begin{array}{l}\text { Evaluar el efecto sobre } \\
\text { la funcionalidad e } \\
\text { independencia }\end{array}$ & $\begin{array}{c}30 \\
\text { Edad: } 7-14\end{array}$ & $\begin{array}{l}2 \text { grupos. } \\
\text { G1: KT; } \\
\text { G2: } \sin K T\end{array}$ & $\begin{array}{c}\text { No efectivo a } \\
\text { largo plazo,pero a } \\
\text { corto plazo mejoraron }\end{array}$ & $\mathrm{II} / 4$ \\
\hline Caneschi WF, et al. ${ }^{25}$ & 2014 & $\begin{array}{l}\text { Evaluar el efecto sobre } \\
\text { la sialorrea }\end{array}$ & $\begin{array}{c}11 \\
\text { Edad: } 5-10\end{array}$ & 1 grupo & Efectivo & $\mathrm{IV} / 2$ \\
\hline Ghalwash AM, et al. ${ }^{28}$ & 2012 & $\begin{array}{l}\text { Evaluar el efecto sobre } \\
\text { el genu recurvatum }\end{array}$ & $\begin{array}{c}14 \\
\text { Edad: } 5-7\end{array}$ & $\begin{array}{l}2 \text { grupos. } \\
\text { G1: } K T \text { + terapia física; } \\
\text { G2: férula de rodilla + } \\
\text { + terapia }\end{array}$ & $\begin{array}{c}\text { No efectivo, } \\
\text { a; pero mejora la } \\
+\quad \text { funcionalidad }\end{array}$ & III $/ 4$ \\
\hline Tello CL , et al. ${ }^{26}$ & 2012 & $\begin{array}{l}\text { Evaluar el efecto sobre } \\
\text { la deglución }\end{array}$ & $\begin{array}{c}10 \\
\text { Edad: } 9 \pm 8,76\end{array}$ & 1 grupo & Efectivo & $\mathrm{IV} / 2+$ \\
\hline Simsek TT, et al. ${ }^{24}$ & 2011 & $\begin{array}{l}\text { Evaluar el efecto sobre } \\
\text { la funcionalidad y } \\
\text { la independencia }\end{array}$ & $\begin{array}{c}31 \\
\text { Edad: } 8 \pm 4\end{array}$ & $\begin{array}{l}2 \text { grupos. } \\
\text { G1: fisioterapia }+K T \\
\text { G2: fisioterapia }\end{array}$ & $\begin{array}{c}\text { No efectivo, pero } \\
\text { mejora la } \\
\text { alineación corporal }\end{array}$ & $\mathrm{III} / 4$ \\
\hline Yasukawa A, et al. ${ }^{29}$ & 2006 & $\begin{array}{l}\text { Evaluar el efecto sobre } \\
\text { la funcionalidad del } \\
\text { miembro superior }\end{array}$ & $\begin{array}{c}15 \\
\text { Edad: } 4-16\end{array}$ & 1 grupo & Efectivo & IV $/ 2$ \\
\hline
\end{tabular}


lo que se puede decir que el VNM es una buena herramienta en el tratamiento de la sialorrea. Dichos estudios reportan que la venda adhesiva actúa como un estímulo constante a través de la inervación cutánea de la región suprahioidea del cuello, que brinda retroalimentación que aporta información perceptiva. Pero es necesario reseñar que, en estos artículos, no se utilizan escalas validadas, sino que miden la frecuencia del babeo, irritación de la piel, olor desagradable, necesidad de limpiar la barbilla, dificultad para la alimentación, necesidad de cambiar el babero y la ropa, y todas ellas son variables muy subjetivas evaluadas por sus familiares o cuidadores. Es necesario que, en futuras investigaciones, se utilicen escalas validadas que intenten objetivar los resultados para conseguir dar mayor validez a las investigaciones y que, si no hay un grupo control con otro tratamiento, el tiempo aisladamente no le otorga validez interna al estudio.

La motricidad fina es un problema que padecen los niños diagnosticados con PCI, debido a la alteración en la posición del miembro superior. En el estudio de Keklicek H et al., ${ }^{27}$ los niños tenían un patrón en el miembro superior de rotación interna, con flexión de codo, el antebrazo en pronación, flexión de muñeca, flexión de los dedos y pulgar alojado en la palma. Los niños, en el estudio, fueron divididos aleatoriamente en dos grupos (un grupo control y otro experimental). Se encontraron diferencias significativas entre ambos grupos en el tiempo en que se realizaba la actividad, por lo que, según los resultados de este estudio, se puede pensar que el VNM es una buena terapia para disminuir la espasticidad y mejorar la propiocepción. El artículo de Yasukawa A et al., ${ }^{29}$ fija su objetivo en medir la eficacia en la funcionalidad de las extremidades superiores debido a que los niños con una mala alineación de hombro tienen mayor dificultad para llevar una vida independiente. Para evaluar los resultados, este estudio utilizó la escala de Melbourne, que mide la calidad de movimiento del miembro superior al realizar alcances, agarres y manipulaciones. Los resultados obtenidos fueron satisfactorios y encontraron una mejoría en el control y en la calidad del movimiento. El VNM puede considerarse como un buen complemento en el tratamiento del control y de la funcionalidad del miembro superior en pacientes con PCI.

Otra alteración que un gran número de niños con PCI espástica desarrollan fácilmente es el genu recurvatum, que se trata de una deformidad en la articulación femorotibial en la que el rango de movimiento supera los $0^{\circ}$ de extensión. Se produce debido a la actividad excesiva de los músculos de la parte posterior y distal de la pierna. ${ }^{30}$ La investigación de Ghalwash AM et al., ${ }^{28}$ intenta medir su efectividad en el control del genu recurvatum, pero no encontraron diferencias estadísticamente significativas entre los dos grupos, por lo que no se puede afirmar que el VNM sea efectivo en esta aplicación. Sí se encontró una mejoría en la funcionalidad motora de los niños medida por la GMFM. Se necesitan investigaciones con una mayor muestra, ya que, en esta, solo participaron catorce niños y es muy difícil con una muestra tan pequeña obtener unos datos concluyentes para determinar si el VNM es efectivo o no.

Las investigaciones de Keklicek $\mathrm{H}$ et al., ${ }^{27}$ Caneschi WF et al., ${ }^{25}$ Tello CL et al., ${ }^{26}$ Yasukawa A et al., ${ }^{29}$ encontraron resultados favorables que indican que el VNM tiene efectividad en el tratamiento de la sialorrea, de la funcionalidad del miembro superior afecto y de la funcionalidad de la mano afecta en niños con PCI. Otras investigaciones, como Kaya Kara $\mathrm{O}$ et al., ${ }^{23}$ no cumplieron con los objetivos propuestos sobre la mejora de la funcionalidad y de la independencia, pero sí se encontraron mejorías a corto plazo. El estudio de Ghalwash AM et al., ${ }^{28}$ no consiguió mostrar la eficacia del VNM para mejorar el genu recurvatum, pero obtuvo resultados satisfactorios en la funcionalidad de los niños. Mientras que la investigación de Simsek TT et al., ${ }^{24}$ no encontró resultados estadísticamente significativos que apoyaran la efectividad del kinesiotape en la funcionalidad motora de los niños con PCI, aunque demostró que mejoraba su alineación corporal.

Son varias las limitaciones encontradas en los estudios realizados hasta ahora. Para que unos resultados puedan ser extrapolables a todos los pacientes con PCI, es necesario que las investigaciones aumenten el número de sujetos utilizados en ellas, para así obtener resultados concluyentes con la suficiente evidencia científica. La media de la muestra de los siete estudios incluidos sobre PCI es de veintidós pacientes, y este número debe ser mayor en futuras investigaciones. Con las series de casos o los casos y controles, cuando no se ha hecho un cálculo del tamaño muestral necesario para mostrar diferencias significativas, no pueden aportar suficiente evidencia científica a los estudios.

El tiempo dedicado a las investigaciones 
también debe ser mayor para poder comprobar la eficacia del vendaje a largo plazo. La única investigación que dedica un tiempo adecuado al estudio es Tello CL, et al., ${ }^{28}$ que duró siete meses. Estos pacientes necesitan mucho tiempo de rehabilitación, ya que sus mejorías son a largo plazo en la mayoría de los casos. Pero si no hay un grupo control con otro tratamiento, el tiempo aisladamente no le otorga validez interna al estudio, por tanto, es difícil comprobar si el VNM es efectivo o no a medio-largo plazo.

Se debe realizar una mejor descripción de la aplicación de esta técnica que se ha llevado a cabo en el estudio para poder determinar si es eficaz en cada uno de los casos en los que se aplica. En esta revisión, solo las investigaciones de Tello CL, et al., ${ }^{26}$ y de Simsek TT, et al., ${ }^{24}$ hacen una buena descripción de la aplicación del VNM.

\section{CONCLUSIÓN}

En la mayoría de los resultados de los estudios incluidos en esta revisión, se han obtenido efectos beneficiosos, pero desde el punto de vista de la evidencia científica y calidad metodológica, no existen estudios concluyentes. Es necesario definir criterios estandarizados que puedan demostrar los efectos otorgados por el VNM, ya que no existe un claro consenso en aspectos esenciales de la técnica, como son la duración que se debe mantener el vendaje o la tensión con la que debe ser aplicado.

\section{REFERENCIAS}

1. Hielkema T, Hadders-Algra M. Motor and cognitive outcome after specific early lesions of the brain- a systematic review. Dev Med Child Neurol 2016;58(4):46-52.

2. Lorente-Hurtado I. La parálisis cerebral. Actualización del concepto, diagnóstico y tratamiento. Pediatr Integral 2007;11(8):687-98.

3. Sanger TD. MovementDisorders in Cerebral Palsy.JPediatr Neurol 2015;13(4):198-207.

4. Pascual JM, Koenigsberger MR. Parálisis cerebral: factores de riesgo prenatales. Rev Neurol 2003;37(3):275-80.

5. Wright M, Wallman L. Cerebral Palsy. En: Campbell SK, Palisano RJ, Orlin MN. Physical therapy for children. 4 ed. Missouri: Elsevier-Sanders; 2012:577-627.

6. Kase K, Wallis J, Kase T. Clinical therapeutic applications of the Kinesiotaping method. 2nd ed. Tokyo: Ken Ikai; 2003.

7. Kahanov L. Kinesio taping: An overview of use with athletes, part II. Athl Ther Today 2007;12(4):5-7.

8. Thelen MD, Dauber JA, Stoneman PD. The clinical efficacy of kinesio tape for shoulder pain: a randomized, double-blinded, clinical trial. J Orthop Sports Phys Ther 2008;38(7):389-95.

9. Cools A, Witvrouw E, Danneels L, et al. Does taping influence electromyographic muscle activity in the scapular rotators in healthy shoulders? Man Ther 2007;7(3):154-62.

10. Halseth T, McChesney JW, DeBeliso M, et al. The effects of Kinesio ${ }^{\mathrm{TM}}$ taping on proprioception at the ankle. J Sports
Sci Med 2004;3(1):1-7.

11. May K. Kinesio tape: A better alternative for many applications. Training \& Conditioning 2008;18:126.

12. Yang SR, Heo SY, Lee HJ. Immediate effects of kinesio taping on fixed postural alignment and foot balance in stroke patients. J PhysTher Sci 2015;27(11):3537-40.

13. Heo SY, Kim KM. Immediate effects of Kinesio Taping on the movement of the hyoid bone and epiglottis during swallowing by stroke patients with dysphagia. J Phys Ther Sci 2015;27(11):3355-57.

14. Lee DH, Kim WJ, Oh JS, et al. Taping of the elbow extensor muscle in chronic stroke patients : comparison between before and after three-dimensional motion analysis. J Phys Ther Sci 2015;27(7):2101-3.

15. ŻukB, Księżopolska-OrłowskaK. Usefulness of Kinesiology Taping method in inflammatory rheumatic illnesses in childhood. Reumatologia 2008;46(6):340-7.

16. Kocyigit F, Turkmen MB, Acar M, et al. Kinesio taping or sham taping in knee osteoarthritis? A randomized, doubleblind, sham-controlled trial. Complement Ther Clin Pract 2015;21(4):262-7.

17. Reyhan AÇ, Dereli EE, Çolak TK. Low back pain during pregnancy and kinesio tape application. J BackMusculoskelet Rehabil 2017;30(3):609-13.

18. Do ES, Park KM, Lee SH. A Study on the Effects of the Kinesio Tape Method on Perimenstrual Discomforts. J Korean Community Nurs 2003;14(3):415-23.

19. Bayrakci Tunay V, Akyüz A, Önal S, et al. Comparison of the instant effects of kinesio and McConnell patellar taping on performance in patellofemoral pain syndrome. Fizyoterapi Rehabilitasyon 2008;19(3):104-9.

20. Briem K, Eythörsdöttir H, Magnúsdóttir RG, et al. Effects of the kinesio tape compared with nonelastic sports tape and the untaped ankle during a sudden inversion perturbation in male athletes. J Orthop Sports Phys Ther 2011;41(5):328-35.

21. Karadag-Saygi E, Cubukcu-Aydoseli K, Kablan N, et al. The role of kinesiotaping combined with botulinumtoxinto reduce plantar flexors spasticity after stroke. Top Stroke Rehabil 2010;17(4):318-22.

22. Espejo-Antúnez L, Apolo-Arenas MD. Revisión bibliográfica de la efectividad del kinesiotaping. Rehabilitación 2011;45(2):148-58.

23. Kaya Kara O, Atasavun Uysal S, Turker D, et al. The effects of Kinesio Taping on body functions and activity in unilateral spastic cerebral palsy: a single-blind randomized controlled trial. Dev Med Child Neurol 2015;57(1):81-8.

24. Şimşek TT, Türkücüoğlu B, Çokal N, et al. The effects of Kinesio $^{(R)}$ taping on sitting posture, functional independence and gross motor function in children with cerebral palsy. Disabil Rehabil 2011;33(21-22):2058-63.

25. CaneschiWF, Paiva CC, Frade RL, etal.Use of elasticbandage associated with speech therapy in the control ofsialorrhea (hypersalivation). Rev CEFAC 2014;16(5):1558-66.

26. López Tello C, Escuder González S, Oliván Blázquez B, et al. Eficacia del kinesiotaping en la sialorrea en niños con necesidades educativas especiales: un ensayo clínico abierto. Fisioterapia 2012;34(6):275-81.

27. Keklicek H, Uygur F, Yakut Y. Effects of taping the hand in children with cerebral palsy. J Hand Ther 2015;28(1):27-33.

28. Ghalwash AM, El-Shennawy SA, Abd-Elwahab MS. Efficacy of adhesive taping in controlling genure curvatum in diplegic children: A pilot study. Egypt J Med Hum Genet 2013;14(2):183-8.

29. Yasukawa A, PatelP, Sisung C. Pilotstudy: Investigating the effects of Kinesio Taping in an acute pediatric rehabilitation setting. Am J Occup Ther 2006;60(1):104-10. 